\title{
The Urokinase Receptor (CD87) Facilitates CD11b/CD18-mediated Adhesion of Human Monocytes
}

\author{
R.G. Sitrin, ${ }^{\star}$ R.F. Todd III, ${ }^{\ddagger}$ H.R. Petty, ${ }^{\S}$ T.G. Brock, ${ }^{*}$ S.B. Shollenberger, ${ }^{*}$ E. Albrecht, ${ }^{\S}$ and M.R. Gyetko* \\ $*$ Pulmonary and Critical Care Medicine Division and ${ }^{\ddagger}$ Hematology/Oncology Division, Department of Internal Medicine, University of \\ Michigan, and Veteran's Administration Medical Center, Ann Arbor, Michigan 48109; and ${ }^{\S}$ Department of Biological Sciences, Wayne \\ State University, Detroit, Michigan 48202
}

\begin{abstract}
Urokinase receptors (UPAR; CD87) form complexes with complement receptor 3 (CR3) (CD11b/CD18), a $\beta 2$ integrin. In this study, we sought to determine if this association modulates the adhesive function of CR3. Both CR3 and UPAR concentrate at the ventral surface of fibrinogenadherent human monocytes, and CR3-uPAR coupling increases substantially upon adhesion to fibrinogen. Pretreatment with anti-uPAR monoclonal antibody reduced adhesion to CR3 counterligands (fibrinogen and keyhole limpet hemocyanin) by $\mathbf{5 0 \%}$, but did not affect adhesion to fibronectin, a $\beta 1$ integrin counterligand. Antisense (AS) oligonucleotides were used to determine if selectively suppressing uPAR expression also modulates CR3 adhesive function. AS-uPAR oligo reduced CR3-dependent adhesion by $43 \pm 9 \%(P<0.01)$, but did not affect CR3-independent adhesion. To determine if the effects of uPAR are mediated through its ligand, monocytes were pre-treated with AS oligo to block uPA expression. Unlike the effects of blocking UPAR expression, AS-uPA oligo increased adhesion by $46 \%$ $(P<0.005)$, and exogenous intact uPA, but not uPA fragments, reversed this effect. We conclude that complex formation with UPAR facilitates the adhesive functions of CR3. This function of UPAR is not dependent upon its occupancy with uPA, which negatively influences adhesion. ( $J$. Clin. Invest. 1996. 97:1942-1951.) Key words: adhesion • monocyte • integrin • macrophage- 1 antigen • urokinase
\end{abstract}

\section{Introduction}

Leukocytes must orchestrate a complex sequence of adherence and locomotive steps as they are recruited to emigrate from the vascular space into sites of inflammation. Under some circumstances, this process is initiated by a phase of loose adhesion, mediated by L-selectin binding to counterligands on the endothelium (1). This is followed by a phase of firm attachment, mediated in part by complement receptor 3 (CR3) $^{1}$ (Mac-1, $\left.\alpha \mathrm{M} \beta 2, \mathrm{CD} 11 \mathrm{~b} / \mathrm{CD} 18\right)$, a $\beta 2$ integrin adhesion protein (1). The binding capacity of CR3 for its counterligands is modulated postsynthetically, causing rapid transitions between high and low binding avidity during the sequential at-

Address correspondence to Robert G. Sitrin, M.D., Pulmonary and Critical Care Medicine Division, 3916 Taubman Center, Box 0360, Ann Arbor, MI 48109-0360. Phone: 313-936-5040; FAX: 313-9365048; E-mail: rsitrin@uv1.im.med.umich.edu

Received for publication 12 July 1995 and accepted in revised form 25 January 1996.

J. Clin. Invest.

(C) The American Society for Clinical Investigation, Inc.

0021-9738/96/04/1942/10 \$2.00

Volume 97, Number 8, April 1996, 1942-1951 tachment and detachment steps necessary for cell movement. The molecular mechanisms controlling CR3 binding capacity are complex and not fully elucidated. Aggregation of CR3 enhances its binding activity (2, 3), and activation signaling through CD14 or chemotaxins can enhance CR3 adhesive function as well $(4,5)$. Although the underlying mechanisms are only partially characterized, the conformation of CR3 is known to change during cellular activation (6). Some stimuli induce phosphorylation of CR3, with variable effects on its function $(7,8)$. CR3 function is profoundly affected by changes in extracellular divalent cations (9) and exogenous factors such as integrin modulating factor-1 (IMF-1), a lipid product of cytokine-stimulated polymorphonuclear neutrophils (PMNs) (10). Finally, CR3 forms complexes with other plasma membrane proteins such as the immunoglobulin receptor FcyR III (CD16) $(11,12)$, and the urokinase receptor (uPAR; CD87) (13-15). These proteins are linked to the plasma membrane by glycosylphosphatidylinositol (GPI) anchors, and thus lack a direct connection to the cell interior. This suggests that uPAR must interact with membrane-spanning proteins like CR3 to successfully transmit activation signals. There is evidence that CR3 is important for normal function of FcyR III $(16,17)$. Likewise, CR3 is required for UPAR to trigger an intracellular calcium flux in response to engaging its ligand, urokinase-type plasminogen activator (uPA) (18). Complex formation between CR3 and UPAR is particularly intriguing, since both proteins serve critical, and perhaps complementary, roles in leukocyte trafficking $(1,19,20)$. The physical association between UPAR and CR3 provides a structural basis for coordinating the functions of these two proteins during cellular movement. In this study, we demonstrate that the in vitro adhesive functions of CR3 are strongly influenced by its association with uPAR and uPA. This suggests that the UPAR and uPA system exerts significant regulatory control over CR3 function in monocyte adhesion, locomotion, and activation.

\section{Methods}

Reagents. High molecular weight urokinase (HMW-uPA; $55 \mathrm{kD}$ ), low molecular weight uPA (LMW-uPA, $33 \mathrm{kD}$ ), and murine anti-human uPA monoclonal antibody (mAb) were obtained from American Diagnostica (Greenwich, CT). Recombinant human uPA amino terminal fragment (uPA-ATF) containing the uPAR-binding region (aa 1-135), but not the active site, was generously provided by Richard $\mathrm{H}$. Simon, M.D., University of Michigan Medical Center. The uPA-

1. Abbreviations used in this paper: AS, antisense; CR3, complement receptor 3; GPI, glycosylphosphatidylinositol; HMW-uPA, high molecular weight uPA; KLH, keyhole limpet hemocyanin; LMW-uPA, low molecular weight uPA; NS, nonsense; oligo, oligonucleotides; RET, resonance energy transfer; rs-uPAR, recombinant soluble uPAR; TRITC, tetramethyl rhodamine thioisocyanate; uPA, urokinase plasminogen activator; uPA-ATF, uPA amino terminal fragment; uPAR, uPA receptor. 
ATF, produced with the Xpress protein expression system in E. coli (Invitrogen, San Diego, CA), was synthesized from the pTrcHis vector as a fusion protein with six histidine residues at the $\mathrm{NH}_{2}$ terminus. The fusion protein was then purified by ProBond metal affinity chromatography (Invitrogen), and the recombinant uPA-ATF (18.7 kD) removed from the $\mathrm{NH}_{2}$-terminal fusion peptide at an enterokinase cleavage site. Recombinant soluble uPAR (CD87) was generously provided by Robert L. Cohen, M.D., University of California, San Francisco. The mouse anti-human uPAR mAb (clone 3B10, $\mathrm{IgG}_{2 \mathrm{a}}$ ), also designated anti-Mo3f, was prepared as previously described, and recognizes an epitope in the UPAR ligand binding region (domain 1) $(21,22)$. An isotype-matched anti-CD38 mAb (Biosource, Camarillo $\mathrm{CA})$ was used as a control for the anti-uPAR mAb. Anti-integrin mAbs included an anti-CD11b mAb (clone 44, IgG2a) (23); and an anti-CD18 mAb (clone TS1/18, IgG1) (24). Where indicated, these $\mathrm{mAbs}$ were conjugated with fluorescein isothiocyanate (FITC) or tetramethyl rhodamine thioisocyanate (TRITC), using standard methods (15). Phorbol myristate acetate (PMA) was obtained from Sigma Chemical Co. (St. Louis, MO).

Purification of monocytes. Monocytes were obtained from peripheral blood of healthy volunteers by density centrifugation (25). Heparinized peripheral blood was diluted 1:1 in PBS with $2.5 \mathrm{mM}$ EDTA and spun through Lymphoprep (GIBCO BRL, Gaithersburg, MD) at $800 \mathrm{~g}, 23^{\circ} \mathrm{C}$, for $25 \mathrm{~min}$. The interphase cells were collected and diluted $1: 1$ in $\mathrm{Ca}^{2+}-\mathrm{Mg}^{2+}$-free $\mathrm{PBS} / 0.1 \%$ bovine serum albumin (BSA), washed, in $\mathrm{PBS} / 0.1 \% \mathrm{BSA} / 1 \%$ gelatin, resuspended in PBS, mixed 1:2 with Percoll (Pharmacia, Piscataway, NJ) in $1.65 \% \mathrm{NaCl}$, and centrifuged at $2000 \mathrm{~g}, 23^{\circ} \mathrm{C}$, for $20 \mathrm{~min}$. The top $1 / 2$ volume was aspirated, and the monocytes washed and resuspended in culture medium. Cell viability was monitored by trypan blue exclusion. The composition of preparations was generally $>90 \%$ monocytes, as determined by differential analysis of Wright/Giemsa-stained cytocentrifuge preparations.

Fluorescence microscopy. Glass slides (Nunc Lab-Tek; Thousand Oaks, CA) were pre-coated with fibrinogen $(5 \mathrm{mg} / \mathrm{ml}$ in PBS; Calbiochem, San Diego, CA) for $1 \mathrm{~h}, 37^{\circ} \mathrm{C}$, washed, and counter-coated for 30 min with $1 \%$ BSA. Monocytes were added to the coated slides $\left(0.75 \times 10^{6}\right.$ per chamber $)$ along with FITC anti-uPAR mAb or FITC anti-CD $11 \mathrm{~b} \mathrm{mAb}$, and PMA $(5 \mathrm{ng} / \mathrm{ml})$ in $150 \mu \mathrm{l}$ of RPMI $+0.01 \% \mathrm{Na}$ azide $/ 0.1 \% \mathrm{BSA}$, and incubated for $30 \mathrm{~min}$ at $37^{\circ} \mathrm{C}$. For dual staining, the cells were first incubated with anti-CD11b mAb, followed sequentially by a phycoerythrin-goat anti mouse $\mathrm{IgG}$, a $1 \%$ mouse serum blocking step, and finally FITC anti-uPAR mAb. The nonadherent cells were gently removed with $\mathrm{PBS}, 37^{\circ} \mathrm{C}$, and fixed for $10 \mathrm{~min}$ with $2 \%$ formaldehyde, $37^{\circ} \mathrm{C}$. The slides were mounted with ProTexx medium (Baxter, Romulus, MI) and examined with a Biorad MRC 600 confocal microscope (Biorad, Hercules, CA).

Immunofluorescence flow cytometry. To measure plasma membrane CR3, cells were suspended in staining buffer (PBS with 1\% BSA and $0.1 \%$ sodium azide, $\mathrm{pH} 7.4$ ), labeled for $30 \mathrm{~min}, 4^{\circ} \mathrm{C}$, with R-phycoerythrin-conjugated anti-CD11b $\mathrm{mAb}$, and nonspecific background staining was measured with R-phycoerythrin-conjugated IgG (Becton-Dickinson, San Jose, CA). Intact (live) cells were selected by gating (log forward angle vs. log right angle light scatter). Immunofluorescence intensity was assessed as a measure of relative antigen expression, using a Coulter Electronics EPICS C flow cytometer (Hialeah, FL) with a logarithmic amplifier. The channel number (log scale) representing the mean fluorescence intensity (major fluorescence peak) was determined from $\sim 5,000$ cells. The corresponding linear fluorescence intensity channel was then calculated from a logarithmic-linear calibration formula. Specific fluorescence intensity was calculated by subtracting background fluorescence, using the linear scale.

Resonance energy transfer (RET) microscopy. For these experiments, monocytes were labeled with FITC-conjugated $\mathrm{F}\left(\mathrm{ab}^{\prime}\right)_{2}$ fragments of anti-CD11b (clone 44) for $30 \mathrm{~min}, 0^{\circ} \mathrm{C}$, followed by labeling with TRITC-conjugated anti-uPAR $\mathrm{F}\left(\mathrm{ab}^{\prime}\right)_{2}$ fragments for $30 \mathrm{~min}$, $0^{\circ} \mathrm{C}$. Cells were adhered to glass coverslips (untreated, or coated with $100 \mu \mathrm{l}$ of $10 \mu \mathrm{g} / \mathrm{ml}$ fibrinogen for $2 \mathrm{~h}, 37^{\circ} \mathrm{C}$ ) for $2 \mathrm{~h}$ at $37^{\circ} \mathrm{C}$. An axio- vert 135 inverted fluorescence microscope (Carl Zeiss Inc., New York, NY) with mercury illumination interfaced to a Perceptics Biovision workstation (Knoxville, TN) was employed for cell examination. The fluorescence images were collected by an intensified CCD camera (model XC-77; Hamamatsu, Japan) and image processor. Narrow bandpass discriminating filter sets were used for FITC (excitation 485/22 nm, emission 530/30 nm) and rhodamine excitation 540/ $20 \mathrm{~nm}$, emission 590/30 nm) (Omega Optical, Brattleboro, VT). Long pass dichroic mirrors of $510 \mathrm{~nm}$ and $560 \mathrm{~nm}$ were used for FITC and TRITC, respectively. For RET microscopy (26), the 485/22 nm narrow bandpass discriminating filter was used for excitation and the $590 / 30 \mathrm{~nm}$ filter was used for emission. The $510 \mathrm{~nm}$ long-pass dichroic mirror was used for RET $(27,28)$. Differential interference contrast (DIC) photomicrographs were collected by using Zeiss polarizers and a CCD camera (model WV-BL200; Panasonic). The background-subtracted digitized images were averaged and stored on hard disk or streaming tape. Images were photographed using a freeze-frame video recorder (Polaroid, Boston, MA). The RET levels of the samples were quantitated using a photon counting apparatus (Photochemical Research Associates, London, Ont) coupled to the microscope (29). The photomultiplier tube (Hamamatsu) was held in a Products-for-Research housing (Danvers, MA) and cooled with a Peltier system interfaced to a constant temperature water bath/circulator (Forma Scientific; Marietta, $\mathrm{OH}$ ). Cells were illuminated individually to ensure that each RET level corresponded to a single cell. Background photon count rates were always taken from an adjacent area on the slide containing no cells, and uniformly represented a small fraction of the total photon count rate. The differences between the photon count rates of the cells and their matched background count rates were recorded as RET intensity levels.

Preparation of substrate-coated culture plates. Polystyrene sterile tissue culture wells ( $16 \mathrm{~mm}$; Costar, Cambridge, MA) were incubated with fibrinogen $\left(5 \mathrm{mg} / \mathrm{ml}\right.$ in PBS) for $2 \mathrm{~h}$ at $4{ }^{\circ} \mathrm{C}$, washed with PBS, and counter-coated with $1 \%$ BSA (6). Wells were also coated with keyhole limpet hemocyanin (KLH; $0.5 \mathrm{mg} / \mathrm{ml}$; Sigma Chemical Co.) or fibronectin $(20 \mu \mathrm{g} / \mathrm{ml}$; Collaborative Research, Bedford, MA) for $2 \mathrm{~h}$, $37^{\circ} \mathrm{C}$, followed by BSA counter-coating and extensive washing with PBS $(30,31)$.

Antisense oligonucleotide inhibition of $U P A, U P A R$ expression. Antisense oligonucleotides (AS-oligos) represented the reverse complement of high specificity sequence regions. The AS-oligos for uPA and UPAR correspond to nucleotides $426-444$ and $720-738$, respectively $(32,33)$. The control oligos consisted of nonsense (NS) oligos with the same base composition as the AS sequences, but in random order. AS and NS oligos were synthesized by the DNA Synthesis Core of the University of Michigan on automated DNA synthesizers, substituting sulfur-for-oxygen at the phosphate moiety. These phosphorothioate oligos are nuclease resistant (34). Cells were incubated with $30 \mathrm{mM}$ AS-oligos for $48 \mathrm{~h}$ at $37^{\circ} \mathrm{C}$. We have previously demonstrated the efficacy and specificity of blocking UPAR and UPA in human monocytes with AS-oligo treatment by showing marked diminution of immunofluorescent staining for the corresponding protein (19). Cell viability exceeding $95 \%$ was confirmed by trypan blue exclusion prior to using AS-oligo treated cells in experiments.

Treatment of monocytes with antibodies, recombinant $u P A R, u P A$ fragments. Immediately after purification, or after pretreatment with oligos, as indicated, $2 \times 10^{6}$ monocytes were dispensed into microcentrifuge tubes and incubated with mAbs, recombinant soluble uPAR (rs-uPAR), or uPA fragments for $30 \mathrm{~min}, 4^{\circ} \mathrm{C}$. Anti-uPAR and antiCD38 mAbs, purified by Protein A affinity chromatography and quantitated by protein content, were added to yield a final concentration of $2.5 \mu \mathrm{g} / \mathrm{ml}$. Anti-CR3 mAbs were used as 1:100 dilutions of ascites fluid. Cells were then pelleted, resuspended in serum-free medium (MAC-SFM; GIBCO BRL) at $37^{\circ} \mathrm{C}$, and immediately added to the substrate-coated wells.

Adhesion assay. Cells were incubated for $1 \mathrm{~h}$ at $37^{\circ} \mathrm{C}$, and nonadherent cells removed by gentle washing. Unless indicated otherwise, the monocytes were stimulated with PMA $(10 \mathrm{ng} / \mathrm{ml})$ as they were 
added to the wells. Adherent cells were quantitated by staining with 2,5-diphenyltetrazolium bromide thiazolyl blue vital dye $(5 \mathrm{mg} / \mathrm{ml}$; Sigma Chemical Co.) for $4 \mathrm{~h}$. The cells were then washed, lysed, and absorbance measured at $550 \mu \mathrm{m}$. For every experiment, some monocytes were reserved, counted with a hemocytometer, and serially diluted aliquots stained in parallel to generate the standard curve to convert absorbances to cell numbers.

Statistics. Comparisons between group means were performed with paired, two-tailed Student's t test, unless otherwise indicated. A $P$ value $\leq 0.05$ was considered statistically significant. Adhesion data were collected as the mean number of adherent cells in duplicate wells. The means \pm SEM represent the compiled data from experiments, each performed with monocytes prepared from separate donors.

\section{Results}

Association of $u P A R$ with $C R 3$. Experiments were first undertaken to demonstrate that uPAR and CR3 interact during monocyte adhesion to fibrinogen-coated surfaces. Confocal microscopy was performed to compare the distribution of uPAR and CR3 on the plasma membrane. As shown in Fig. 1 $I$, both UPAR and CR3 are locally concentrated at the plane of cell-matrix contact. In both cases, immunostaining revealed a patchy, granular pattern. As the focal plane moved away from the plane of adhesion, both uPAR and CR3 progressively exhibited dimmer and more homogenous staining. Co-localization of UPAR and CR3 at the adhesion plane of fibrinogen-adherent monocytes was further demonstrated, using dual-labeling for CD11b and uPAR and confocal microscopy (Fig. 1II). The merged images from the two fluorochromes demonstrates substantial co-localization of CR3 and UPAR at the plane of adhesion to fibrinogen.

To ascertain the molecular proximity relationships between UPAR and CR3, we have performed RET microscopy $(15,26)$. Fig. 2 shows DIC, FITC, TRITC, and RET photomicrographs of monocytes adherent to glass coverslips, uncoated or coated with fibrinogen. Using the photon counting system to obtain quantitation of UPAR-CR3 coupling, low but detectable levels of energy transfer were observed for cells adhered to glass. As demonstrated previously in neutrophils, the presence of resonance energy transfer indicates the close proximity of uPAR and CR3 in the membrane $(15,18)$. Adhesion to a fibrinogen-coated surface induced a substantial increase in en-
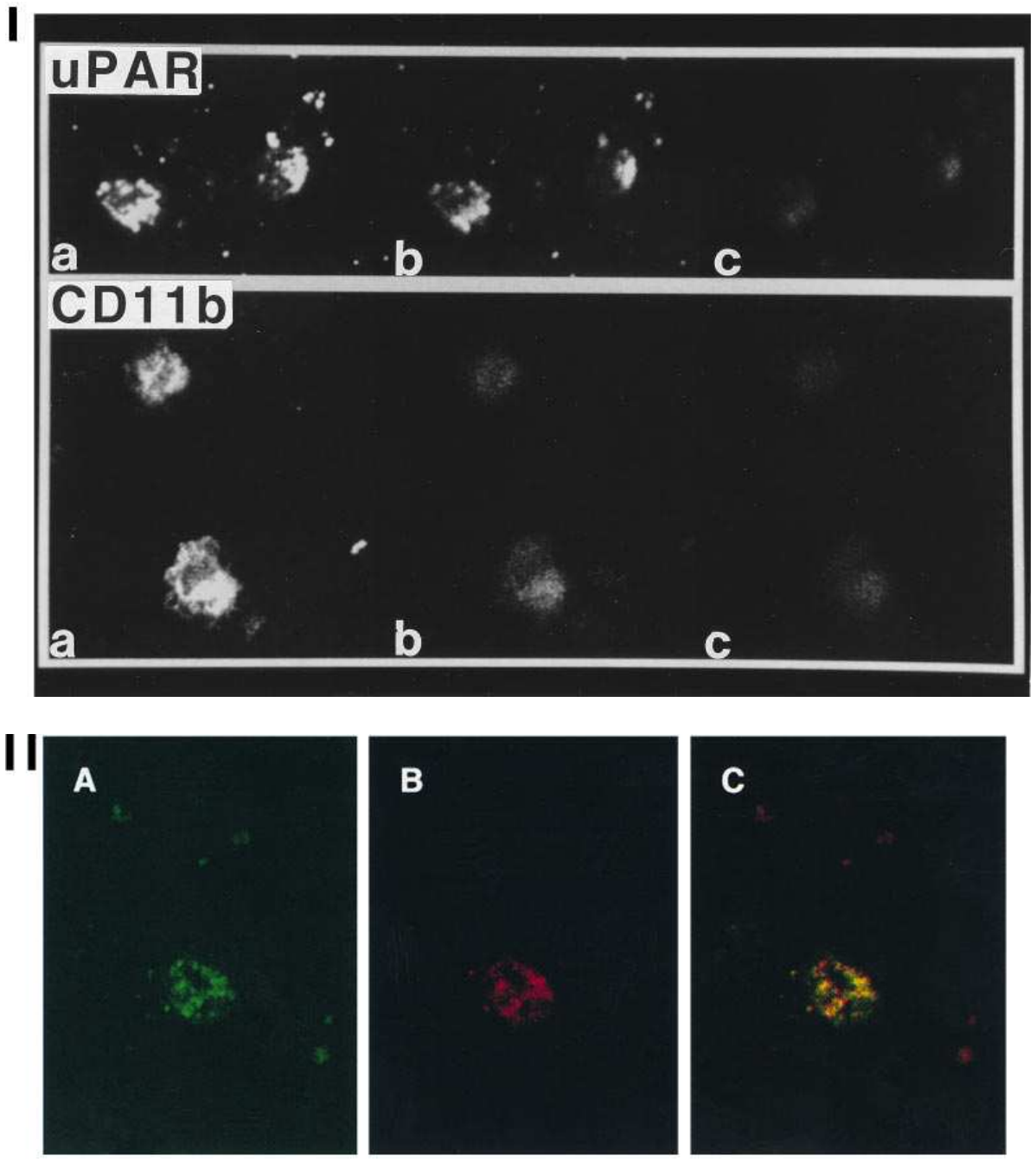

Figure 1. (I) Co-localization of uPAR and CR3, demonstrated by confocal microscopy of monocytes adherent to fibrinogen-coated glass. Cells were imaged with a $60 \times$ oil immersion lens and a zoom of 2.0. Transverse optical sections (pinhole aperture of 5; nominal section thickness of $\sim 0.5 \mu \mathrm{m}$ ) were obtained at the level of attachment to the slide, and at $0.5 \mu \mathrm{m}$ steps above this adhesion plane. An image of each optical section was created by averaging six one-second scans. Cells stained with non-immune IgG controls yielded completely negative results. ( $U p$ per panel) Cells labeled with FITC antiuPAR, with images representing the plane of contact with fibrinogen $(a), 1 \mu \mathrm{m}$ above the adhesion plane $(b)$, and $2 \mu \mathrm{m}$ above the adhesion plane (c). (Lower panel) Cells labeled with FITC anti$\mathrm{CD} 11 \mathrm{~b}$, with images representing the plane of contact with fibrinogen $(a), 1 \mu \mathrm{m}$ above the adhesion plane $(b)$, and $2 \mu \mathrm{m}$ above the adhesion plane (c). (II) Colocalization of UPAR and CR3, demonstrated by dual-staining and merged images obtained by confocal microscopy. Monocytes adherent to fibrinogencoated glass slides were first labeled with anti-CD $11 \mathrm{~b} \mathrm{mAb}$ and PE-conjugated goat anti-mouse IgG. The cells were then washed, blocked with $1 \%$ mouse serum, and then labeled with FITC anti-uPAR $\mathrm{mAb}$. The dual-labeled cells were scanned by optical sectioning at the adhesion plane by confocal microscopy, as described in A. uPAR $(A)$ and CD11b $(B)$ were visualized simultaneously by collecting separately the FITC emission (using a 514-nm bandpass filter combined with a

526-nm-long pass dichroic reflector) and the PE emission (using a 560-nm long pass filter followed by a $585 \mathrm{~nm}$ bandpass filter), respectively. Yellow regions in $(C)$, obtained by overlaying images $(A)$ and $(B)$, indicate co-localization of uPAR and CR3 at the adhesion plane. 

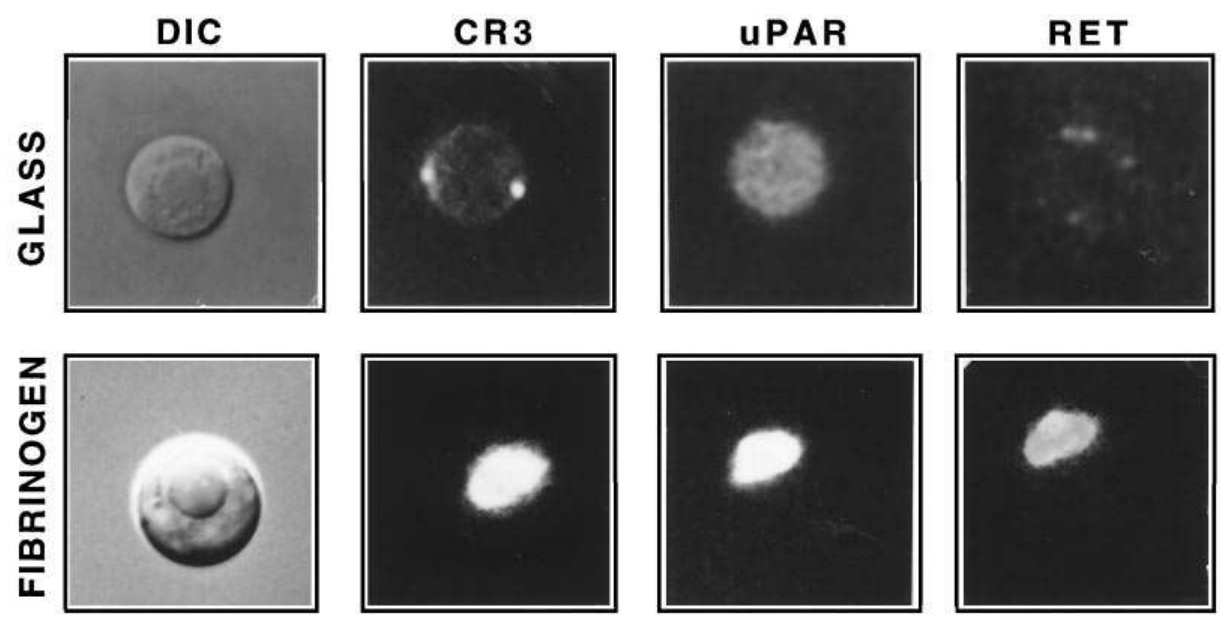

Figure 2. Resonance energy transfer microscopy of monocytes adherent to glass or fibrinogen. Cells were doublelabeled with fluorochrome-conjugated $\mathrm{mAbs}$ and examined by differential interference contrast (DIC), fluorescence of the FITC-conjugated anti-CD11b mAb, and the TRITCconjugated anti-uPAR $\mathrm{mAb}$, and resonance energy transfer (RET), as shown. In the representative cells shown, uPAR and CD11b are co-dis-

Adhesion Surface Resonance Energy Transfer

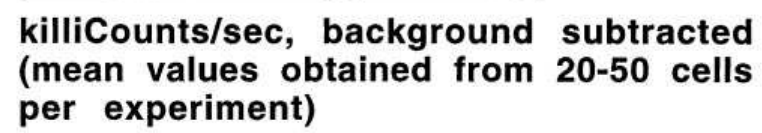
tributed on the cell surfaces. RET was demonstrated by excitation of the FITC with the $485 / 22 \mathrm{~nm}$ beam, and measuring 590/30 $\mathrm{nm}$ emission from the TRITC-labeled anti-uPAR mAb.

Glass

$40 \pm 7$

Fibrinogen

$198 \pm 6$
( $p<0.001$ vs. adhesion to glass) $\mathrm{n}=3$ experiments adherent monocytes, while substantial RET fluorescence is seen for monocytes adherent to fibrinogen. The accompanying table shows quantitative uPAR-CD11b RET data pooled from 3 experiments.

ergy transfer, indicating a marked increase in molecular coupling between uPAR and CR3.

Anti-uPAR antibody suppresses CR3-mediated monocyte adhesion. To determine if UPAR influences the function of
CR3, monocytes were treated with anti-uPAR mAb or an isotype-matched control mAb (anti-CD38). PMA-stimulated adhesion to fibrinogen and KLH, CR3 counterligands $(30,35)$, was then quantitated. To determine the extent to which adhe-
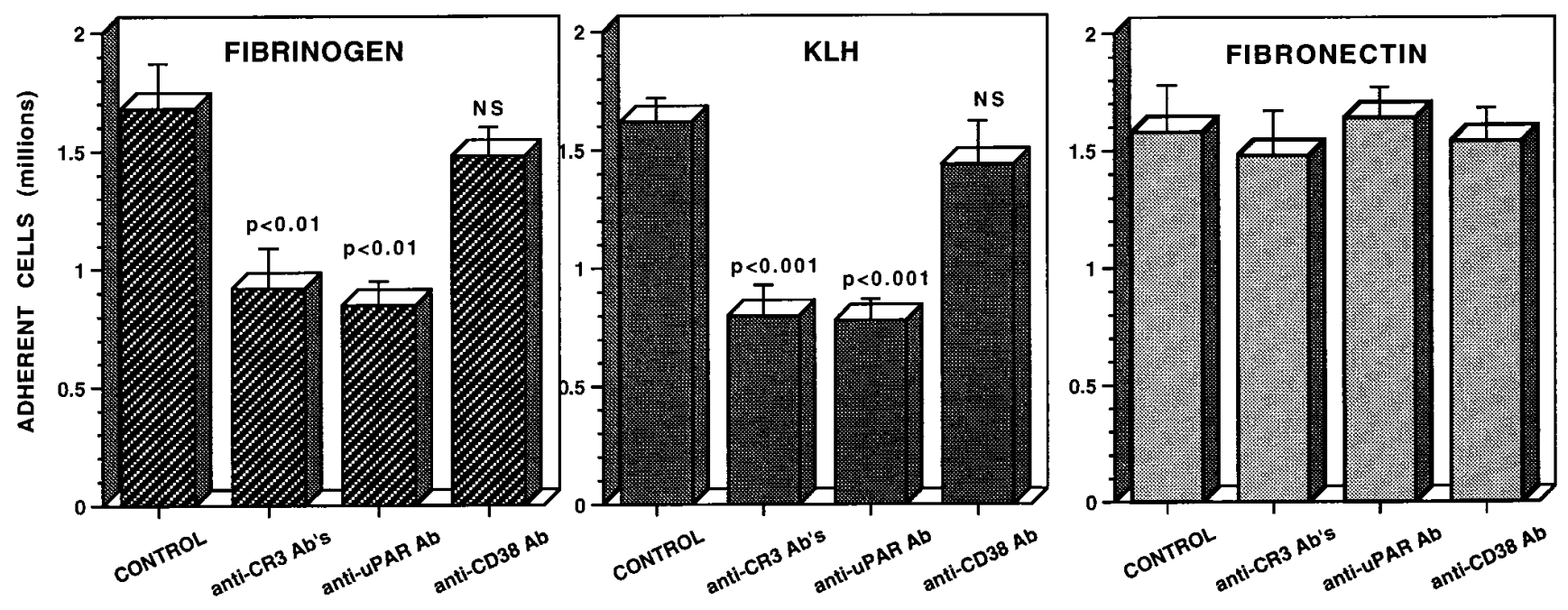

Figure 3. Effects of anti-CR3 mAbs (anti-CD11b+anti-CD18), uPAR mAb, and control mAb (anti-CD38) on PMA-stimulated monocyte adhesion to fibrinogen and KLH (counterligands for CR3), and fibronectin (a $\beta 1$ integrin counterligand). The number of adherent cells, out of $2 \times$ $10^{6}$ plated per $16 \mathrm{~mm}$ well, is expressed in millions. The anti-CR3 mAbs suppressed adhesion to fibrinogen and KLH by $50 \%$, but had no effect on adhesion to fibronectin. The anti-uPAR mAb suppressed adhesion to fibrinogen and KLH to the same extent as anti-CR3 mAb, but did not affect adhesion to fibronectin. The control mAb did not affect adhesion to any of the substrates. 
sion to these surfaces is CR3-dependent, the cells were pretreated in parallel with a combination of an anti-CD11b mAb and anti-CD18 mAb. The anti-CR3 mAb combination suppressed adhesion to both fibrinogen and KLH by $50 \%(P<$ 0.001 ; Fig. 3), verifying substantial CR3 dependence. In preliminary experiments, anti-CD11b $\mathrm{mAb}$ alone was nearly as effective in blocking adhesion as the combination of mAbs (not shown). Pre-treating with the anti-uPAR mAb reduced adhesion to both fibrinogen and KLH by approximately $50 \%$ $(P<0.001)$, while the control $\mathrm{mAb}$ did not affect adhesion at all (Fig. 3). The anti-uPAR mAb only affected adhesion to CR3 counterligands, since adhesion to fibronectin, a $\beta 1$ integrin counterligand, was completely unaffected by the anti-CR3 $\mathrm{mAb}$ combination, anti-uPAR mAb, and the anti-CD38 control. Monocytes were then treated with the anti-CR3 or antiuPAR mAbs alone or in combination to determine if blocking CR3 and uPAR has additive effects on adhesion to fibrinogen. In this series of experiments, blocking either CR3 or uPAR once again significantly reduced adhesion (to $63.1 \pm 6.2 \%$ and $61.0 \pm 6.5 \%$ of untreated controls, respectively; $P<0.01$ ), and the $\mathrm{mAb}$ combination did not reduce adhesion any further $(66.7 \pm 5.5 \%$ of untreated control). These findings suggest that uPAR and CR3 mediate adhesion through a common mechanism, consistent with the observed formation of CR3-uPAR complexes. Thus, the anti-uPAR mAb specifically suppresses CR3-dependent adhesion, and does not have other nonspecific effects on monocyte adhesion.

Experiments were next performed to verify that the antiuPAR mAb was not affecting CR3-mediated adhesion by a quantitative effect on CR3 expression. Monocytes were pretreated with either anti-uPAR or anti-CD38 control mAbs according to the same protocol used in the adhesion experiments, immunolabeled with a R-phycoerythrin-anti-CD11b $\mathrm{mAb}$, (or R-phyco IgG to measure background fluorescence), and analyzed by flow cytometry. The specific fluorescence in-

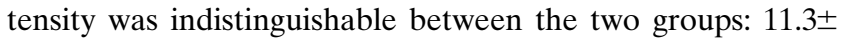
4.9 (anti-uPAR mAb) and 11.6 \pm 4.7 (anti-CD38 mAb), as measured in linear arbitrary units. Because anti-uPAR mAb did not affect CD11b levels in parallel with adhesion, it is concluded that uPAR affects the function of CR3 rather than the number of CR3 sites available to interact with counterligands.

Antisense oligonucleotide blockade of UPAR expression decreases CR3-mediated monocyte adhesion. The close associa- tion between CR3 and uPAR creates the possibility that the anti-uPAR mAb reduced monocyte adhesion by sterically interfering with the interaction between CR3 and its counterligand, rather than UPAR specifically modulating the conformation or function of CR3. Therefore, we sought to determine if selectively blocking $\mathrm{UPAR}$ expression with antisense oligonucleotides would be sufficient to reduce CR3-mediated adhesion to KLH. Monocytes were either pretreated with antisense- or nonsense-oligos for $48 \mathrm{~h}$ in serum-free medium (MAC-SFM), and then allowed to adhere to KLH-coated plates in the presence of PMA. Adhesion of control monocytes pre-incubated in medium alone for $48 \mathrm{~h}$ was measured with and without the anti-CR3 mAbs. After culture for $48 \mathrm{~h}$, it was apparent that there was considerable inter-donor variability in the degree to which adhesion to KLH remained CR3-dependent. This may be explained by reduced CR3 levels in monocytes cultured for prolonged periods in vitro (36), although this was not specifically examined in the present study. Of nine subjects studied, the monocytes of five remained dependent on CR3 for adhesion to KLH, as evidenced by $\geq 30 \%$ suppression of adhesion by the anti-CR3 mAbs, while monocytes from the remaining four subjects were not affected by the anti-CR3 mAbs. As shown in Fig. 4, antisense blockade of uPAR expression significantly reduced adhesion to KLH where CR3

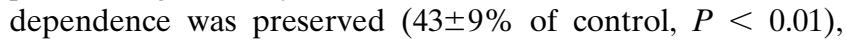
while anti-sense uPAR oligo had no effect at all when adhesion to KLH was CR3-independent. In all these experiments, the control (nonsense) oligo did not significantly affect adhesion, eliminating possible sequence-nonspecific effects of phosphorothioate oligonucleotides on adhesion (37). It was also verified by immunofluorescence flow cytometry that ASuPAR oligo did not reduce the level of CD11b expression (Table I). In keeping with the effects of anti-uPAR mAb, prior treatment with AS-uPAR oligo had no effect on monocyte adhesion to fibronectin (not shown). Thus, we conclude that reducing uPAR expression is sufficient to impair the adhesive function of CR3, and this cannot be explained by nonspecific effects of the oligos, or by modulation of adhesive functions unrelated to CR3. The specificity of suppressing adhesion with AS-uPAR oligo is also demonstrated by reversing the adhesive defect with recombinant uPAR (see below).

Exogenous recombinant $u P A R$ substitutes for endogenous $u P A R$ in modulation of CR3-mediated adhesion. Experi-
CR3-DEPENDENT ADHESION

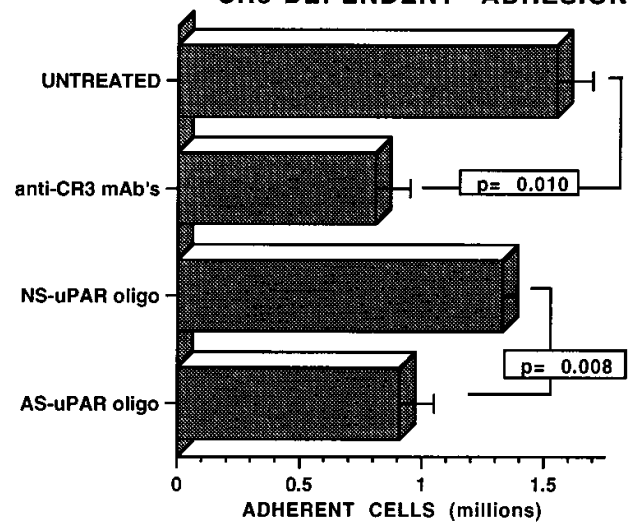

CR3-INDEPENDENT ADHESION

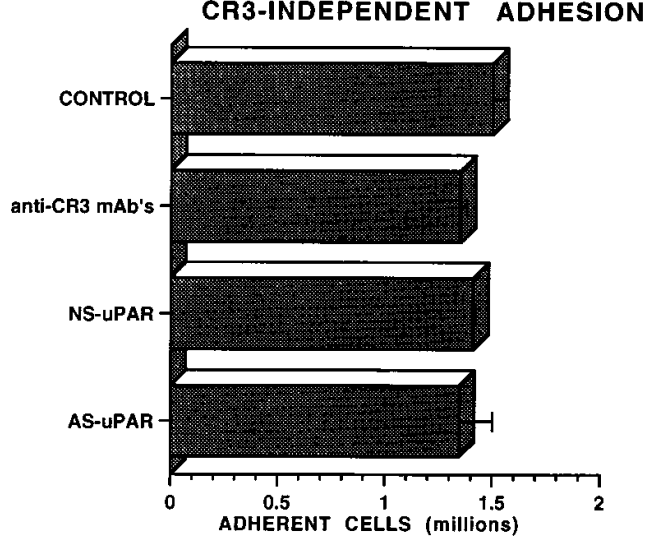

Figure 4. Effects of blocking uPAR expression with antisense (AS)-oligonucleotide on PMA-stimulated monocyte adhesion to KLH. Data are expressed as in Figure 2. After pre-treatment with AS-oligo, nonsense (NS) control oligo, or medium alone (untreated), KLH adhesion remained CR3dependent ( $\geq 30 \%$ suppression with anti-CR3 mAbs) in 5 of 9 subjects (left), and was no longer CR3-dependent in the remaining 4 (right). In the monocytes retaining CR3-

dependent adhesion, AS-uPAR significantly reduced adhesion relative to the NS-oligo treated control $(P<0.008)$, while NS oligo had no significant effect, relative to untreated controls. CR3-independent adhesion was completely unaffected by either AS-oligo or NS-oligo. 
Table I. Effects of Antisense Oligonucleotide Blockade on CD11b Expression

\begin{tabular}{lcc}
\hline \multicolumn{3}{c}{ CD11b specific fluorescence intensity* } \\
\hline No prior treatment & Antisense uPAR oligo & Control uPAR oligo \\
\hline $10.7 \pm 0.6$ & $10.0 \pm 0.9$ & $11.5 \pm 3.9$ \\
No prior treatment & Antisense uPA oligo & Control uPA oligo \\
\hline $12.6 \pm 1.5$ & $8.6 \pm 4.1$ & $11.0 \pm 3.0$
\end{tabular}

*Assessed by immunofluorescence flow cytometry 45 minutes after stimulation with PMA $(5 \mathrm{ng} / \mathrm{ml})$. Data expressed in arbitrary units of fluorescence intensity, linear scale with background subtracted; mean \pm $\mathrm{SEM}, n=3$. None of the oligonucleotides significantly changed CD11b fluorescence intensity, relative to cells without prior treatment (all $P>$ $0.05)$.

ments were next performed to determine if interaction with domains 1-3 of uPAR modulates CR3-mediated adhesion, or alternatively, if the GPI anchor linking UPAR to the plasma membrane is necessary as well. A requirement for the GPI anchor would suggest that this region is required for interacting with CR3, or that uPAR uses the GPI anchor to trigger generation of secondary messengers that affect the "inside-out" signaling that modulates the adhesive function of CR3. To test these concepts, monocytes were rendered deficient in endogenous UPAR by pre-treatment with AS-uPAR oligo, as described above. Cells were then incubated with $2.5 \mu \mathrm{g} / \mathrm{ml} \mathrm{re-}$ combinant soluble (rs)-uPAR (full length, lacking the GPI anchor) for $30 \mathrm{~min}, 4^{\circ} \mathrm{C}$, washed, and resuspended in fresh medium. PMA-stimulated adhesion to fibrinogen-coated plates was then measured. The rs-uPAR reconstituted the adhesion of AS-uPAR oligo-treated cells to a level statistically indistinguishable from untreated control cells (Fig. 5). Control experiments verified that rs-uPAR treatment of AS-uPAR oligo-

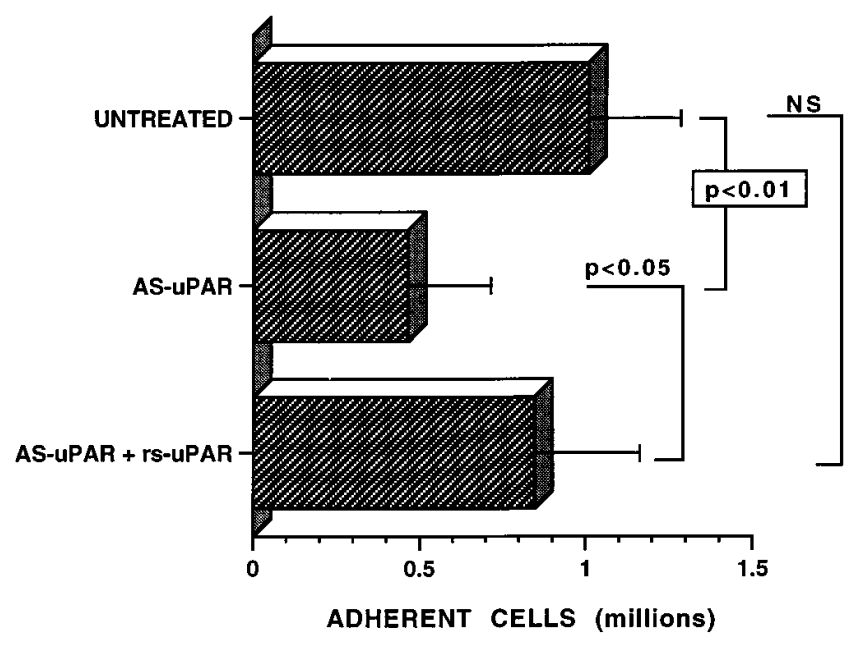

Figure 5. Effects of recombinant soluble uPAR (rs-uPAR) on monocytes rendered uPAR-deficient by pre-treatment with antisense (AS)-uPAR oligo. PMA-stimulated adhesion to fibrinogen is shown, and data are expressed as in Fig. 2. AS-uPAR reduced adhesion to fibrinogen by $46 \%(P<0.01)$, just as it reduced adhesion to KLH (shown in Fig. 3). Adding rs-uPAR to AS-uPAR oligo-treated cells significantly increased adhesion $(P<0.05)$ to a level statistically indistinguishable from untreated cells incubated in medium alone. treated monocytes does not affect adhesion to fibronectin (102 $\pm 6.5 \%$ control). In addition, freshly isolated monocytes (with no AS-oligo pre-treatment) were incubated with rs-uPAR as above, and adhesion to fibrinogen-coated plates was measured. Under these conditions, rs-uPAR did not affect CR3mediated adhesion, either in the absence or presence of PMA (106 \pm 7 and $107 \pm 18 \%$ control, respectively). These results indicate that exogenous rs-uPAR can restore CR3-mediated adhesion of uPAR-depleted cells, but the quantity of uPAR expressed by unstimulated monocytes is sufficient to permit optimal CR3 adhesive function. In addition, complex formation with uPAR is sufficient to affect CR3-mediated adhesion, even in the absence of its GPI anchor. It remains possible, however, that signaling through the GPI anchor can also contribute to regulating CR3 function, since the role of cooperative signaling through uPAR may have been minimized in these experiments by using saturating concentrations of rs-uPAR.

The role of $u P A R$-associated $U P A$ in modulating CR3mediated adhesion. One of the possible mechanisms by which uPAR could modulate CR3 function is by facilitating an interaction between uPA and CR3. In this scenario, uPA may bind to CR3 and alter its adhesive properties. Alternatively, membrane-associated uPA or plasmin catalytic activities may affect the structure of CR3 or other associated proteins. Previous work has shown that the $\alpha$ chain of guinea pig CR3 is sensitive to plasmin, but not uPA (38). Finally, uPA may affect CR3 function if its binding to UPAR generates intracellular second messenger molecules that affect the "inside-out" activation of CR3. The anti-uPAR mAb used in previous experiments poorly displaces uPA from its binding site, suggesting that uPAR and CR3 interact directly, but this does not eliminate the possibility that receptor-associated uPA also participates in this process. Therefore, it was necessary to specifically examine whether uPA modulates CR3-mediated adhesion in a way that could account for some of the effects of uPAR. First, endogenous production of UPA was suppressed by pretreating monocytes for $48 \mathrm{~h}$ with AS-uPA oligo, along with nonsense (NS)-uPA control oligo. Adhesion to fibrinogen was then assessed. Because preliminary experiments indicated that blocking uPA expression increased adhesion, these experiments were performed without added PMA, which increases adhesion to maximal levels. As shown in Fig. 6, blocking uPA expression increased adhesion to fibrinogen by $46 \%$, relative to untreated controls $(P<0.005)$. This was not due to a nonspecific effect of the oligo, since the NS-uPA oligo had a suppressive effect on adhesion which was statistically insignificant. Moreover, neither the AS-oligo nor control oligo affected the level of CR3 expression (Table I). Enhanced adhesion in response to AS-uPA was specific for a CR3 counterligand, since adhesion to fibronectin was unaffected under identical circumstances (Fig. 6). In addition, the anti-CR3 mAbs suppressed adhesion of AS-uPA pre-treated cells to fibrinogen by 55.2 \pm $1.4 \%$ (not shown). These results suggest that endogenously produced uPA has a suppressive effect on the adhesive function of CR3, and further, the reduced CR3-dependent adhesion resulting from interfering with UPAR cannot be explained simply by modulating receptor-associated uPA. In the next series of experiments, monocytes were pre-treated with AS-uPA oligo to suppress endogenous uPA production. Cells were then incubated for $30 \mathrm{~min}, 4^{\circ} \mathrm{C}$, with $10 \mu \mathrm{M}$ concentrations of purified intact, high molecular weight uPA (HMW-uPA), low molecular weight uPA (LMW-uPA), which contains an intact cat- 

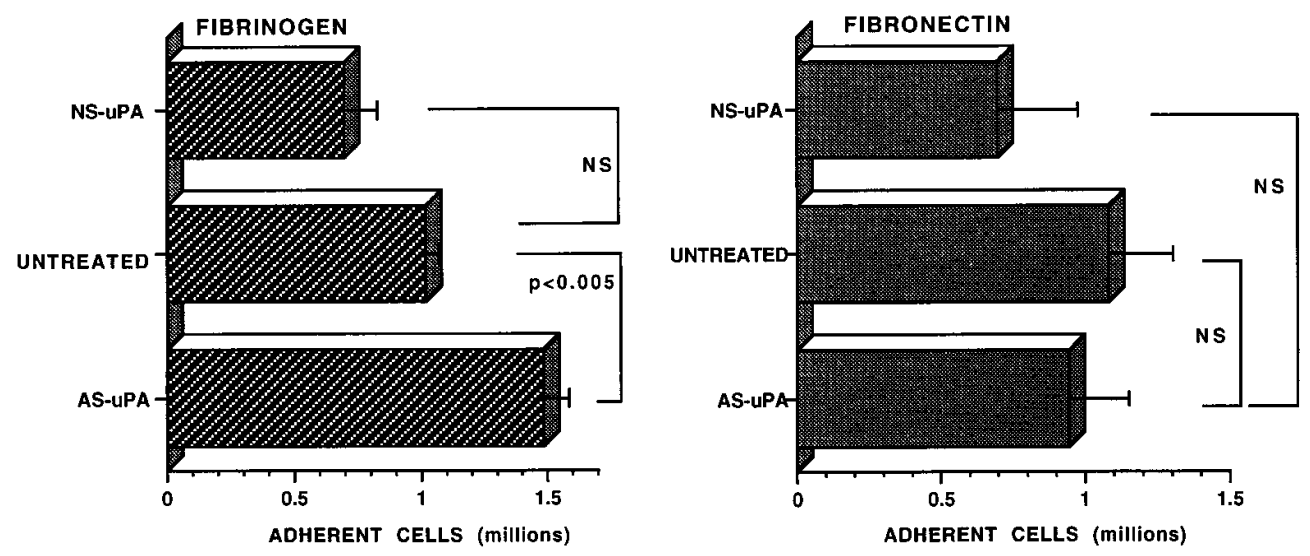

Figure 6. Effects of blocking uPA expression with antisense (AS)-uPA oligonucleotide, nonsense (NS)-oligo, or medium alone (untreated). Adhesion to fibrinogen was measured (without PMA stimulation), and data are expressed as in Fig. 3. ASuPA oligo pre-treatment increased adhesion by $46 \%(P<$ 0.005), while the control NSoligo had an insignificant suppressive effect. Adhesion of ASuPA oligo treated cells was twice that of the NS-oligo control. By contrast, neither the NSuPA nor the AS-uPA oligo significantly affected adhesion to fibronectin. alytic domain but no uPAR-binding domain, and UPA-amino terminal fragment (uPA-ATF), which binds to uPAR but lacks the catalytic domain. This concentration was chosen because previous work demonstrated that $10 \mu \mathrm{M}$ uPA-ATF was sufficient to maximally displace endogenous uPA from the surface of uPAR-bearing tumor cells, indicating that this represented a saturating concentration (not shown). Saturating available uPAR with HMW-uPA suppresses monocyte adhesion to fibrinogen by $57 \%$ relative to AS-uPA oligo-treated controls $(P<0.005$; Fig. 7$)$. By contrast, an equimolar concentration of uPA-ATF has a small though statistically significant suppressive effect $(90.3 \%$ of control, $P<0.05)$. The LMWuPA had a small and variable suppressive effect which did not reach statistical significance ( $80 \%$ control, $P=0.23$ ). These results support the specificity of blocking uPA with AS-oligo,

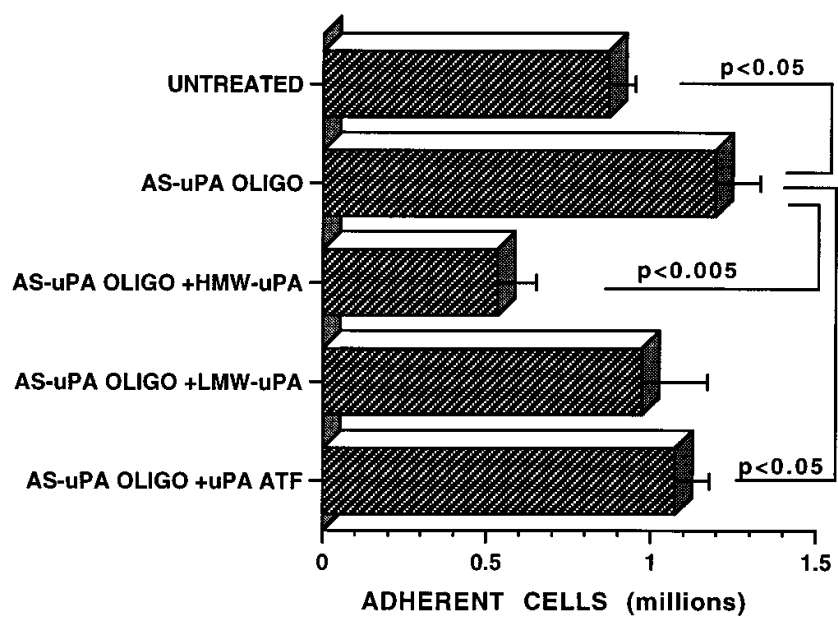

Figure 7. Effects of exogenous uPA or uPA fragments on AS-uPA oligo treated monocytes. Adhesion to fibrinogen was measured (without PMA stimulation), and data are expressed as in Fig. 3. ASuPA oligo significantly increased adhesion relative to untreated controls, as shown in Fig. 6. Exogenous HMW-uPA reduced adhesion by $50 \%(P<0.005)$. Exogenous uPA-amino terminal fragment (ATF) had a small but significant suppressive effect on adhesion. By contrast, exogenous low molecular weight (LMW)-uPA, bearing only the catalytic domain, did not significantly affect adhesion. since adding the natural product (HMW-uPA) reverses the effect. In addition, they indicate that intact UPA, containing both uPAR-binding and catalytic domains is required to interfere with CR3 adhesive function. Combined with the results shown in Fig. 3 and 4 and Table I, these observations lead us to conclude that the uPAR-CR3 interaction is critically dependent on the association between UPA and uPAR. While UPAR is required for CR3 adhesive function, $\mathrm{UPAR}$ occupied with intact UPA do not facilitate CR3-mediated adhesion optimally, and in fact, may negatively influence adhesion. Thus, the adhesive properties of CR3 are maximized by interaction with unoccupied UPAR.

\section{Discussion}

CR3 has a firmly established role in migration and activation of mononuclear phagocytes and PMNs (1). Numerous models of leukocyte-mediated tissue injury have shown that blocking CR3 function not only interferes with leukocyte adhesion, but also subsequent tissue infiltration and injury of target cells (1). The absence of $\beta 2$ integrins in the leukocyte adhesion deficiency syndrome has verified that defective CR3 function causes severely dysfunctional leukocytes and impaired host defenses in vivo (39). We have recently demonstrated that the urokinase receptor (UPAR; CD87) is necessary for chemotaxis of human monocytes and PMNs in vitro $(19,40)$. In this study, we show that uPAR modulates the adhesive function of CR3, offering a plausible mechanism by which UPAR can participate in leukocyte trafficking and activation.

Prior studies have demonstrated a close physical association between CR3 and uPAR in human PMNs, using co-capping and resonance energy transfer $(15,18)$. More recently, coprecipitation studies of monocyte lysates have shown that uPAR exists in large complexes with CR3 and LFA-1 (13). The present study confirms by confocal microscopy that CR3 and uPAR co-distribute on monocytes adhered to a CR3 counterligand (fibrinogen) (Fig. 1). Under these conditions, both proteins are highly concentrated at the plane of cellular contact with the substratum, enhancing the opportunity for functional interaction between the two proteins. Resonance energy transfer studies confirm that CR3 and UPAR are not 
only co-distributed on the cell surface, but are in very close physical proximity (41) (Fig. 2). The resolution limit of resonance energy transfer is within $7 \mathrm{~nm}$, while co-localization by conventional immunofluorescence microscopy has a Rayleigh limit of $\geq 180 \mathrm{~nm}$ (26). Molecular coupling of uPAR with CR3, as demonstrated by quantitative energy transfer (Fig. 2), is dramatically increased by engagement of a CR3 counterligand (fibrinogen). At present, it is not known whether conformational changes in CR3 or propagation of activation signals through CR3 are responsible for this effect.

Direct linkages between CR3 and cytoskeletal elements can be induced with leukocyte activation, consistent with the observed redistribution of CR3 with adhesion to a ligandcoated surface $(27,42,43)$. Prior studies have shown that uPAR also clusters at adhesion foci and co-distributes with cytoskeletal elements $(44,45)$. However, uPAR, anchored to the plasma membrane by a GPI moiety, has no cytoplasmic domain to link directly with the cytoskeleton. Thus, it is plausible that redistribution of UPAR to the membrane-fibrinogen interface is driven by its association with CR3. In support of this view, uPAR capping is defective in neutrophils with defective $\beta 2$ integrins (46). Co-localization of uPAR with CR3 is true not only for adhesion, but also leukocyte chemotaxis. Clustering of uPAR occurs at the leading edge of migrating monocytes (19). Likewise, studies of neutrophils in a chemotactic gradient show that newly expressed CR3 localizes to the leading edge of migration and then moves toward the uropod and trailing filopodia (47).

Adhesion to CR3 counterligands was shown to be dependent upon UPAR, based on the suppressive effects of antiuPAR mAb (Fig. 3). The close proximity of CR3 and uPAR raised the possibility that the anti-uPAR $\mathrm{mAb}$ could block adhesion by sterically interfering with ligand binding sites or inducing conformational changes on CR3. However, this mechanism of action was excluded by showing that selectively blocking uPAR expression with AS-uPAR oligo also effectively suppresses CR3-dependent adhesion, duplicating the results seen with the anti-uPAR mAb (Figs. 4 and 5). The present data strongly suggest that $\mathrm{UPAR}$ acts to facilitate the function of CR3, rather than having any independent effects on adhesion, or acting as an alternative binding site for the CR3 counterligands. This conclusion is based on several observations. First, maximal suppression of adhesion produced by anti-uPAR mAb was not additive to that of the anti-CR3 mAbs, suggesting that the antibodies were working through redundant mechanisms. Further, anti-uPAR mAb did not affect adhesion to fibronectin (Fig. 3), and blocking UPAR expression with AS-oligo only reduced CR3-dependent adhesion (Fig. 4). Finally, prior studies have shown that purified uPAR does not bind directly to fibrinogen (48). While it is possible that there is an overlapping interaction between UPAR and CD11c/CD18, which also binds fibrinogen (49), this is an unlikely explanation for our findings, since CD11c/CD18 expression is quite low in freshly isolated monocytes (36), and consequently, the dominant fibrinogen-binding protein is CR3. Determining the precise mechanisms by which uPAR modulates CR3 function will certainly be an important avenue for future investigation.

It is clear that UPAR facilitates CR3 function by mechanisms that are independent of its role as an anchorage site for uPA. In fact, adhesion was significantly increased by blocking uPA expression with AS-uPA oligo (Fig. 6). This would sug- gest that unoccupied UPAR is the ideal partner protein for optimizing CR3 adhesive function. Only intact HMW-uPA was able to reverse the effects of blocking uPA expression, since the effect could not be duplicated by either uPA fragment (uPA-ATF or LMW-uPA; Fig. 7). Thus, we can conclude that uPA does not reduce CR3 adhesive function simply by occupying the ligand binding region of UPAR, nor does it function solely by actions of the catalytic domain. Further work will be necessary to define more precisely the elements of uPA structure responsible for influencing CR3-dependent adhesion. It will also be important to determine how CR3 function is affected by factors that regulate UPAR occupancy, such as activation-induced changes in UPAR binding affinity or UPA synthesis $(50,51)$. Ambient levels of SERPIN-class PA inhibitors may also affect this process, since uPA-PAI-1 complexes are rapidly internalized, and may produce a net reduction in uPAR occupancy $(52,53)$.

One of the most notable features of CR3 is the plasticity of its function once it is expressed on the plasma membrane, enabling the cells to adapt to inflammatory stimuli with very rapid changes in CR3-mediated functions (1). This property of CR3 is also likely to be important in cellular locomotion, where portions of the cell membrane moving from the lamellopodium to the uropod must successively engage and disengage contact sites on the substratum. The post-synthetic mutability of CR3 function is multifactorial. Aggregation of CR3 can increase avidity for ligands (3). In addition, CR3 phosphorylation, extracellular cations, and IMF-1 can modulate CR3 function (7-10). The present findings add uPAR-CR3 complex formation and changes in UPAR occupancy to the list of mechanisms for inducing rapid changes in CR3 binding avidity. It remains to be determined how activation-related postsynthetic modifications of CR3 affect its interactions with uPAR/uPA. In our preliminary studies (not shown), adhesion of unstimulated monocytes to fibrinogen was reduced by antiuPAR mAb, although PMA stimulation certainly enhanced adhesion and accentuated the effect of blocking uPAR.

An important role for uPAR and UPA in cellular movement was first established with numerous studies of leukocytes and malignant cells, demonstrating that pericellular plasmin formation permits cells to digest and penetrate extracellular matrix barriers $(20,50)$. Plasmin, a broad spectrum endopeptidase, can cleave many ECM glycoproteins and proteoglycans directly, and in addition, can activate latent matrix metalloproteinases (54-56). The clustering and co-localization of CR3 with UPAR would be a strategic mechanism of concentrating pericellular uPA and plasmin activities at sites of firm adhesion. This would enable the cells to disengage from sites of firm adhesion to $\mathrm{CR} 3$ counterligands, and may also provide a mechanism for limiting the degree of ECM degradation necessary for cells to initiate migration through these barriers. In this scheme, CR3 would assume a primary role in directing the orientation and distribution of pericellular plasminogen activation. More recent work has demonstrated that UPAR and uPA do far more than regulating pericellular uPA and plasmin activities. We have shown that $\mathrm{UPAR}$ expression is necessary for chemotaxis of human monocytes and PMN's, independently of its ability to bind uPA $(19,40)$. The results of the present study suggest that one of the functions of UPAR in chemotaxis may be to permit CR3 to function in adhesion and cytoskeletal rearrangements necessary for cellular locomotion. It is still premature to speculate as to the importance of uPAR-CR3 inter- 
actions with other physiologically relevant counterligands. Monocyte adhesion to complex structures such as endothelial cells in vitro requires the compounded effects of multiple adhesion molecules $(57,58)$. Evidence is already emerging to indicate that UPAR forms complexes with several other adhesion proteins, including LFA- 1 , and $\beta 1$ and $\beta 3$ integrins, and the effects of uPAR on their adhesive functions are presently unknown $(13,59)$. Thus, the effect of uPAR on monocyteendothelial binding may represent the aggregate of interactions with several partner proteins, and the role of UPAR on CR3 function may be very difficult to isolate in this context.

Other potential roles for uPAR and UPA have been demonstrated. PAI-1, which binds avidly to vitronectin in ECM, can bind to uPAR-anchored uPA, and thus may serve as an intermediary to bind uPAR-bearing cells to vitronectin (60). Finally, recent studies have shown that UPAR itself can be induced to function as a vitronectin adhesion receptor, and binding uPA to UPAR enhances this process $(48,61)$. By contrast, the present study demonstrates that binding uPA to uPAR diminishes CR3-mediated adhesion. Together, these findings raise the intriguing possibility that UPAR occupancy selectively modulates the ability of UPAR to interact with ECM, enhancing binding to some ECM constituents (vitronectin), while reducing binding avidity to others (CR3 counterligands). The enzymatic activities of membrane-associated uPA and plasmin are likely to strongly influence adhesion as well. This may occur by degrading the constituents of ECM that would serve as adhesion counterligands. In addition, the $\alpha$ chain of guinea pig CR3 is plasmin sensitive (38), and uPAR itself is partially degraded by uPA and plasmin (62).

The potential importance of uPAR-CR3 interactions extends beyond cellular adhesion and migration. Adhesion, and particularly CR3 engagement, is an important co-factor for activation of mononuclear phagocytes in vitro (63). Likewise, recent studies have shown that UPAR can mediate cellular activation and differentiation signals $(13,18)$. Engagement of UPAR with uPA can prime PMNs for superoxide production and an increase in intracellular calcium concentrations (18). CR3 was shown to be an obligate co-factor for uPA-uPAR engagement in generating this calcium flux. Thus, interactions between the uPAR-uPA system and CR3 may modulate the ability of both proteins to influence activation signaling.

In summary, we have shown that plasma membrane urokinase receptors participate in monocyte adhesion by modulating the function of CR3. Receptor occupancy with HMW-uPA diminishes the capacity of UPAR to facilitate CR3 function. Combined with previous observations (18), this work establishes that the functions of CR3 and uPAR are mutually interdependent, and therefore, interactions between CR3 and the uPAR-uPA system can potently affect monocyte trafficking and activation.

\section{Acknowledgments}

The authors thank Kris Weber for her assistance with flow cytometry, and Ikuko Mizukami and Laura Mayo-Bond for preparation of monoclonal antibodies.

This work was supported by National Institutes of Health grants HL-39672 and HL-53283 (R.G. Sitrin), CA-42246 and CA-39064 (R.F. Todd), AI/CA 27409 (H.R. Petty), a Merit Review Grant of the Veteran's Administration (M.R. Gyetko), and a grant from the American Heart Association of Michigan (M.R. Gyetko).

\section{References}

1. Beekhuizen, H., and R. vanFurth. 1993. Monocyte adherence to human vascular endothelium. J. Leukocyte Biol. 54:363-378.

2. Detmers, P., S. Wright, E. Olsen, B. Kimball, and Z. Cohn. 1987. Aggregation of complement receptors on human neutrophils in the absence of ligand. J. Cell Biol. 105:1137-1145.

3. Hermanowski-Vosatka, A., P. Detmers, O. Gotze, S. Silverstein, and S. Wright. 1988. Clustering of ligand on the surface of a particle enhances adhesion to receptor-bearing cells. J. Biol. Chem. 263:17822-17827.

4. Wright, S., R. Ramos, A. Hermanowski-Vosatka, P. Rockwell, and P. Detmers. 1991. Activation of the adhesive capacity of CR3 on neutrophils by endotoxin: dependence on lipopolysaccharide binding protein and CD14. $J$. Exp. Med. 173:1281-1286.

5. Detmers, P., S. Lo, E. Olsen-Egbert, A. Walz, M. Baggiolini, and Z. Cohn. 1990. Neutrophil activating protein 1/interleukin 8 stimulates the binding activity of the leukocyte adhesion receptor CD11b/CD18 on human neutrophils. J. Exp. Med. 171:1155-1162.

6. Diamond, M., and T. Springer. 1993. A subpopulation of Mac-1 (CD11b/ CD18) molecules mediates neutrophil adhesion to ICAM-1 and fibrinogen. $J$. Cell Biol. 120:545-556.

7. Buyon, J., S. Slade, J. Reibman, S. Abramson, M. Philips, G. Weissmann, and R. Winchester. 1990. Constitutive and induced phosphorylation of the $\alpha$ and $\beta$-chains of the CD11/CD18 leukocyte integrin framily: relationship to adhesion-dependent function. J. Immunol. 144:191-197.

8. Chatila, T., R. Geha, and M. Arnaout. 1989. Constitutive and stimulusinduced phosphorylation of CD11/CD18 leukocyte adhesion molecules. J. Cell Biol. 109:3435-3444.

9. Altieri, D. 1991. Occupancy of CD11b/CD18 (Mac-1) divalent ion binding site(s) induces leukocyte adhesion. J. Immunol. 147:1891-1898.

10. Hermanowski-Vosatka, A., J. VanStrijp, W. Swiggard, and S. Wright. 1992. Integrin modulating factor-1: a lipid that alters the function of leukocyte integrins. Cell. 68:341-352.

11. Zhou, M., R. Todd III, J. vandeWinkel, and H. Petty. 1993. Cocapping of the leuko-adhesin molecules complement receptor type 3 and lymphocyte function-associated antigen-1 with Fcg receptor III on human neutrophils. Possible role of lectin-like molecules. J. Immunol. 150:3030-3041.

12. Sehgal, G., K. Zhang, R. Todd III, L. Boxer, and H. Petty. 1993. Lectinlike inhibition of immune complex receptor-mediated stimulation of neutrophils: Effects on cytosolic calcium release and superoxide production. J. Immunol. 150:4571-4580.

13. Bohuslav, J., V. Horejsi, C. Hansmann, J. Stöckl, U. Weidle, O. Majdic, I. Barke, W. Knapp, and H. Stockinger. 1995. Urokinase plasminogen activator receptor, $\beta 2$ integrins, and src-kinases within a single receptor complex of human monocytes. J. Exp. Med. 181:1381-1390.

14. Petty, H., and R. Todd III. 1993. Receptor-receptor interactions of complement receptor type 3 in neutrophil membranes. J. Leuk. Biol. 54:492-494.

15. Xue, W., A. Kindzelskii, R. Todd III, and H. Petty. 1994. Physical association of complement receptor type 3 and urokinase-type plasminogen activator receptor in neutrophil membranes. J. Immunol. 152:4630-4640.

16. Detmers, P., D. Zhou, and D. Powell. 1994. Different signaling pathways for CD18-mediated adhesion and Fc-mediated phagocytosis. J. Immunol. 153:2137-2145.

17. Krauss, J., H. Poo, W. Xue, L. Mayo-Bond, R. Todd III, and H. Petty. 1994. Reconstitution of antibody-dependent phagocytosis in fibroblasts expressing Fc $\gamma$ Receptor IIIB and the complement receptor type 3. J. Immunol. 153:1769-1777.

18. Cao, D., I. Mizukami, B. Garni-Wagner, A. Kindzelskii, R. Todd III, L. Boxer, and H. Petty. 1995. Human urokinase-type plasminogen activator primes neutrophils for superoxide anion release: possible roles of complement receptor type III and calcium. J. Immunol. 154:1817-1829.

19. Gyetko, M., R. Todd III, C. Wilkinson, and R. Sitrin. 1994. The urokinase receptor is required for monocyte chemotaxis in vitro. J. Clin. Invest. 93: 1380-1387.

20. Kirchheimer, J.C., and H.G. Remold. 1989. Endogenous receptorbound urokinase mediates tissue invasion of human monocytes. J. Immunol. 143:2634-2639.

21. Min, H., R. Semnani, I. Mizukami, K. Watt, R. Todd III, and D. Liu. 1992. cDNA for Mo3, a monocyte activation antigen, encodes the human receptor for urokinase plasminogen activator. J. Immunol. 148:3636-3642.

22. Mizukami, I., B. Garni-Wagner, L. deAngelo, M. Liebert, A. Flint, D. Lawrence, R. Cohen, and R. Todd III. 1994. Immunologic detection of the cellular receptor for urokinase plasminogen activator. Clin. Immunol. Immunopathol. 71:96-104.

23. Dana, N., B. Styrt, J. Griffin, R. Todd III, M. Klempner, and M. Arnaout. 1986. Two functional domains in the phagocyte membrane glycoprotein Mo1 identified with monoclonal antibodies. J. Immunol. 137:3259-3263.

24. Sanchez-Madrid, F., J. Nagy, E. Robbins, P. Simon, and T. Springer. 1983. A human leukocyte differentiation antigen family with distinct $\alpha$ subunits and a common $\beta$ subunit: The lymphocyte function-associated antigen (LFA-1), the C3bi complement receptor (OKM-1/Mac-1), and the p150,95 molecule. J. Exp. Med. 158:1785-1803. 
25. Vissers, M., S. Lester, and J. Fantone. 1988. Rapid purification of human peripheral blood monocytes by centrifugation through Ficoll-Hypaque and Sepracell-MN. J. Immunol. Methods. 110:203-207.

26. Uster, P., and R. Pagano. 1986. Resonance energy transfer microscopy: observations of membrane-bound fluorescent probes in model membranes and living cells. J. Cell Biol. 103:1221-1234.

27. Zhou, M., R. Todd III, and H. Petty. 1991. Detection of transmembrane linkages between immunoglobulin or complement receptors and the neutrophil's cortical microfilaments by resonance energy transfer microscopy. J. Mol. Biol. 218:263-268.

28. Zhou, M., H. Poo, R. Todd III, and H. Petty. 1992. Surface-bound immune complexes trigger transmembrane proximity between complement receptor type 3 and the neutrophil's cortical microfilaments. J. Immunol. 148:35503553.

29. Maher, R., D. Cao, L. Boxer, and H. Petty. 1993. Simultaneous calciumdependent delivery of neutrophil lactoferrin and reactive oxygen metabolites to erythrocyte targets: evidence supporting granule-dependent triggering of superoxide release. J. Cell Physiol. 156:226-234.

30. Shappell, S., C. Toman, D. Anderson, A. Taylor, M. Entman, and C. Smith. 1990. Mac-1 (CD11b/CD18) mediates adherence dependent hydrogen peroxide production by human and canine neutrophils. J. Immunol. 144:27022711.

31. Suchard, S., M. Burton, V. Dixit, and L. Boxer. 1991. Human neutrophil adherence to thrombospondin occurs through a CD11/CD18-independent mechanism. J. Immunol. 146:3945-3952.

32. Riccio, A., G. Grimaldi, P. Verde, G. Sebastio, S. Boast, and F. Blasi. 1985. The human urokinase-plasminogen activator gene and its promoter. $\mathrm{Nu}$ cleic Acids Res. 13:2759-2771.

33. Roldan, A.L., V.V. Cubellis, L.R. Lund, K. Danø, E. Appella, and F. Blasi. 1990. Cloning and expression of the receptor for human urokinase plasminogen activator, a central molecule in cell surface, plasmin dependent proteolysis. EMBO (Eur. Mol. Biol. Organ.) J. 9:467-474.

34. Stein, C., and J. Cohen. 1989. Phosphorothioate oligonucleotide analogues. In Antisense inhibitors of gene expression. CRC Press, Inc, Boca Raton, FL.

35. Wright, S., J. Weitz, A. Huang, S. Levin, S. Silverstein, and J. Loike. 1988. Complement receptor type three (CD11b/CD18) of human polymorphonuclear leukocytes recognizes fibrinogen. Proc. Natl. Acad. Sci. USA. 85:77347738.

36. Myones, B., J. Dalzell, N. Hogg, and G. Ross. 1988. Neutrophil and monocyte cell surface p150,95 has iC3b-receptor (CR4) activity resembling CR3. J. Clin. Invest. 82:640-651.

37. Watson, P., R. Pon, and R. Shiu. 1992. Inhibition of cell adhesion to plastic substratum by phosphorothioate oligonucleotide. Exp. Cell Res. 202: 391-397.

38. Remold-O'Donnell, E., and K. Lewandrowski. 1981. Macrophage surface component gp160: sensitivity to plasmin and other proteases. J. Immunol. 128:1541-1544.

39. Albelda, S., C. Smith, and P. Ward. 1994. Adhesion molecules and inflammatory injury. FASEB J. 8:504-512.

40. Gyetko, M., R. Sitrin, J. Fuller, R. Todd, and T. Standiford. 1995. Function of the urokinase receptor (CD87) in PMN chemotaxis. J. Leukocyte Biol. 58:533-538.

41. Szöllösi, J., S. Damjanovich, S. Mulhern, and L. Tron. 1987. Fluorescence energy transfer and membrane potential measurements monitor dynamic properties of cell membranes: a critical review. Prog. Biophys. Mol. Biol. 49:6587.

42. Graham, I., H. Gresham, and E. Brown. 1989. An immobile subset of plasma membrane CD11b/CD18 (Mac-1) is involved in phagocytosis of targets recognized by multiple receptors. J. Immunol. 142:2352-2358.

43. Pavalko, F., and S. LaRoche. 1993. Activation of human neutrophils induces an interaction between the integrin $\beta 2$-subunit (CD18) and the actin binding protein a-actinin. J. Immunol. 151:3795-3807.

44. Ciambrone, G., and P. McKeown-Longos. 1992. Vitronectin regulates the synthesis and localization of urokinase-type plasminogen activator in HT1080 cells. J. Biol. Chem. 267:13617.

45. Takahashi, K., K. Ikeo, T. Gojobori, and M. Tanifuji. 1990. Local function of urokinase receptor at the adhesion contact sites of a metastatic tumor cell. Thromb. Res. Suppl. X:55-61.

46. Kindzelskii, A., W. Xue, R. Todd, L. Boxer, and H. Petty. 1994. Aberrant capping of membrane proteins on neutrophils from patients with leukocyte adhesion deficiency. Blood. 83:1650-1655.

47. Francis, J., R. Todd III, L. Boxer, and H. Petty. 1989. Sequential expression of cell surface C3bi receptors during neutrophil locomotion. J. Cell Physiol. 140:519-523.

48. Wei, Y., D. Waltz, N. Rao, R. Drummond, S. Rosenberg, and H. Chapman. 1994. Identification of the urokinase receptor as an adhesion receptor for vitronectin. J. Biol. Chem. 269:32380-32388.

49. Loike, J., B. Sodeik, L. Cao, S. Leucona, J. Weitz, P. Detmers, S. Wright, and S. Silverstein. 1991. CD11c/CD18 on neutrophils recognizes a domain at the $\mathrm{N}$ terminus of the Aa chain of fibrinogen. Proc. Natl. Acad. Sci. USA. 88:1044-1048.

50. Vassalli, J.-D., A. Wohlwend, and D. Belin. 1992. Urokinase-catalyzed plasminogen activation at the monocyte/macrophage cell surface: a localized and regulated proteolytic system. Curr. Topics Microbiol. Immunol. 181:65-86.

51. Picone, R., E.L. Kajtaniak, L.S. Nielsen, N. Behrendt, M.R. Mastronicola, M.V. Cubellis, M.P. Stoppelli, S. Pedersen, K. Dan $\varnothing$, and F. Blasi. 1989. Regulation of urokinase receptors in monocytelike U937 cells by phorbol ester phorbol myristate acetate. J. Cell Biol. 108:693-702.

52. Olson, D., J. Pöllänen, G. Høyer-Hansen, E. Rønne, K. Sakaguchi, T. Wun, E. Appella, K. Danø, and F. Blasi. 1992. Internalization of the urokinaseplasminogen activator inhibitor type-1 complex is mediated by the urokinase receptor. J. Biol. Chem. 267:9129-9133.

53. Cubellis, M., T. Wun, and F. Blasi. 1990. Receptor-mediated internalization and degradation of urokinase is caused by its specific inhibitor PAI-1. EMBO (Eur. Mol. Biol. Organ.) J. 9:1079-1085.

54. Chapman, H.A., O.L. Stone, and Z. Vavrin. 1984. Degradation of fibrin and elastin by intact human alveolar macrophages in vitro. J. Clin. Invest. 73 : 806-815.

55. Mochan, E., and T. Keler. 1984. Plasmin degradation of cartilage proteoglycan. Biochim. Biophys. Acta. 800:312-315.

56. Murphy, G., and A. Doherty. 1992. The matrix metalloproteinases and their regulation. Am. J. Respir. Cell Mol. Biol. 7:120-125.

57. Carlos, T., A. Dobrina, R. Ross, and J. Harlan. 1990. Multiple receptors on human monocytes are involved in adhesion to cultured human endothelial cells. J. Leukocyte Biol. 48:451-456.

58. Carlos, T., N. Kovach, B. Schwartz, M. Rosa, B. Newman, E. Wayner, C. Benjamin, L. Osborn, R. Lobb, and J. Harlan. 1991. Human monocytes bind to two cytokine-induced adhesive ligands on cultured human endothelial cells endothelial-leukocyte adhesion molecule-1 and vascular cell adhesion molecule-1. Blood. 77:2266-2271.

59. Xue, W., B. Garni-Wagner, R. Todd, and H. Petty. 1995. Colocalization of UPAR with $\beta 1$ and $\beta 3$ integrins on tumor cells adherent to specific extracellular matrices. Proceedings of the 9th International Congress of Immunology. 3208 .

60. Waltz, D., L. Sailor, and H. Chapman. 1993. Cytokines induce urokinase-dependent adhesion of human myeloid cells. A regulatory role for plasminogen activator inhibitors. J. Clin. Invest. 91:1541-1552.

61. Waltz, D., and H. Chapman. 1994. Reversible cellular adhesion to vitronectin linked to urokinase receptor occupancy. J. Biol. Chem. 269:1474614750.

62. Høyer-Hansen, G., E. Rønne, H. Solberg, N. Behrendt, M. Ploug, L. Lund, V. Ellis, and K. Danø. 1992. Urokinase plasminogen activator cleaves its cell surface receptor releasing the ligand-binding domain. J. Biol. Chem. 267: 18224-18229.

63. Fan, S., and T. Edgington. 1993. Integrin regulation of leukocyte inflammatory functions: CD11b/CD18 enhancement of the tumor necrosis factor- $\alpha$ responses of monocytes. J. Immunol. 150:2972-2980. 